\title{
La neurofibromatose 1
}

\section{Pierre Wolkenstein}

\section{ADRESSE}

P. Wolkenstein : service de dermatologie et réseau NF-Mondor, Inserm U. 421, Université Paris XII, Hôpital Henri-Mondor, 51, avenue du Maréchal-de-Lattre-de-Tassigny,

La neurofibromatose 1 (NF1) est une maladie autosomique dominante qui atteint environ 1 individu sur 4000 . Le gène NF1 situé sur le chromosome 17 en q11.2 est constitué d'un domaine d'environ 335 kilobases et code pour une protéine de 2818 acides aminés, la neurofibromine. C'est un gène suppresseur de tumeur. Les mutations ont été identifiées tout au long du gène $N F 1$ sans réel point chaud. Au sein de la même famille dans laquelle les individus ont hérité d'une même mutation NF1, les malades ont des différences phénotypiques marquées allant d'une forme bénigne à une forme grave. Les complications tumorales, neurofibromes plexiformes, tumeurs malignes des gaines nerveuses, tumeurs du système nerveux central, sont causes de morbidité et de mortalité au cours de la neurofibromatose 1. Un certain nombre de données suggèrent que l'inactivation de NF1 est associée à une hyperactivité de Ras dans les cellules tumorales. Cette hyperactivité de Ras semble contribuer à la prolifération cellulaire anormale et permet d'envisager des cibles thérapeutiques.

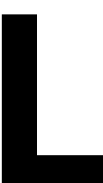

a neurofibromatose 1 (NF1) est une maladie autosomique dominante qui atteint environ 1 individu sur 4000 [1]. En 1882, Von Recklinghausen, pathologiste allemand titulaire de la chaire de Strasbourg, décrivit les tumeurs survenant au cours de cette maladie [2]. Il dénomma neurofibromes ces tumeurs qui se développent à partir des nerfs, et neurofibromatose la maladie au cours de laquelle ces tumeurs sont multiples. Le caractère transmissible de la maladie fut démontré en 1918 et le gène localisé au chromosome 17 en 1987 [3]. Entre ces deux dates, la confusion nosologique fut grande, car les différents auteurs ne distinguaient pas la NF1 (ou neurofibromatose périphérique) de la NF2 (ou neurofibromatose centrale). NF1 et NF2 sont maintenant des maladies reconnues comme distinctes cliniquement, génétiquement et pathologiquement. Les tumeurs de la NF1 sont des neurofibromes, les tumeurs de la NF2 sont des schwannomes. Cette distinction nosologique a été confirmée par le clonage de deux gènes différents situés, respectivement, sur le chromosome 17 et le chromosome 22.

Le gène $N F 1$, situé en $17 q 11.2$, est constitué d'environ 335 kilobases. Son transcrit est approximativement 
de 12 kilobases et code pour une protéine de 2818 acides aminés, la neurofibromine $[4,5]$. La seule fonction reconnue aujourd'hui de la neurofibromine est son interaction avec Ras, par laquelle elle exerce un contrôle négatif de la voie de signalisation Ras/Raf/MAPK [6, 7]. NF1 a trois variants d'épissage majeurs. Cet épissage incorpore de petits segments peptidiques de fonction inconnue [6]. NF1 est régi par un promoteur peu puissant, ce qui conduit à un niveau d'expression faible dans la plupart des cellules des mammifères. Les niveaux d'expression les plus élevés sont retrouvés dans le système nerveux central [8]. Cela est corrélé au développement de troubles cognitifs et de tumeurs cérébrales au cours de la NF1. La localisation intracellulaire de la neurofibromine est dictée par l'interaction avec Ras, qui est ancré dans la partie interne de la membrane cellulaire.

Les mutations du gène $N F 1$ ont été rapportées chez plusieurs centaines de malades [9]. La grande majorité d'entre elles conduit à une inactivation de la neurofibromine. Malheureusement, il n'y a pas de points chauds de mutation et les polymorphismes intragéniques sont donc utilisés pour effectuer des analyses de liaison pour le diagnostic anténatal, dans les cas familiaux de NF1.

Le gène $N F 1$ a été conservé tout au long de l'évolution, de la levure jusqu'aux mammifères. Son activité biologique est, en effet, prépondérante dans les voies de signalisation intracellulaire. La présence quasi constante de neurofibromes multiples chez les malades atteints de NF1, mais aussi d'autres néoplasies, signe son caractère suppresseur de tumeur, la survenue de difficultés d'apprentissage son rôle dans le développement cognitif, les dysplasies osseuses congénitales au cours de la NF1 et les modèles animaux, son intervention dans le développement embryonnaire. Les rôles de $N F 1$ sont donc variés, et le développement de modalités thérapeutiques efficaces passent par leur meilleure compréhension. Dans cette revue, nous explorerons l'épidémiologie de la NF1, les bases moléculaires de la variabilité de son expression clinique, et le rôle de la neurofibromine dans la voie Ras.

\section{Épidémiologie de la NF1}

\section{Prévalence et pénétrance}

La prévalence de la NF1 a été estimée entre une naissance sur 2190 et une naissance sur $7800[10,11]$. Cette prévalence n'a été mesurée que dans les populations dites "caucasiennes» et japonaises. Cependant, il n'y pas d'arguments pour penser que celle-ci pourrait être affectée par les différences ethniques. La pénétrance des mutations NF1 est complète (ou quasi complète) chez l'adulte. Il n'a pas été observé de cas de non pénétrance dans plus de 200 familles étudiées sur plusieurs générations. Les rares cas de non pénétrance rapportés ne sont appuyés ni sur les critères diagnostiques reconnus, ni sur des recherches de mutation ou de liaison. Néanmoins, même si la pénétrance est virtuellement complète à l'âge adulte, la plupart des manifestations de la maladie augmentent avec l'âge. Ainsi est-elle apparente à l'âge de 8 ans chez la grande majorité des individus atteints [12].

\section{Mutations du gène NF1}

La NF1 est une maladie autosomique dominante [13]. Il n'y a pas d'hétérogénéité génétique et tous les malades atteints de NF1 typique ont une mutation au même locus. Tous les individus atteints sont hétérozygotes, aucun exemple convaincant d'homozygotes NF1 n'ayant été rapporté en dépit de la grande prévalence de la maladie et de la consanguinité de certaines familles. L'homozygotie pour l'homologue $N F 1$ de la souris étant létale $[14,15]$, il est probable qu'au moins un allèle NF1 fonctionnel est nécessaire pour le développement fotal précoce.

Une histoire familiale de NF1 est présente dans environ la moitié des cas. La pénétrance du gène étant complète, la moitié des cas de NF1 correspondent donc à des néo-mutations. Les taux estimés de ces néo-mutations varient de 1/7800 à 1/23000. Malgré l'imprécision de cette fourchette, on peut donc considérer que $N F 1$ a l'un des taux de mutation le plus élevé chez l'homme. Plusieurs explications ont été avancées. La grande taille du gène pourrait être un facteur impor- tant, mais n'est certainement pas la seule explication $[16,17]$. Les palindromes, les éléments symétriques, et les séquences répétées sont associés avec la survenue préférentielle d'insertions et de délétions dans certains gènes [18]. De nombreuses délétions et insertions surviennent dans la NF1, mais aussi des substitutions. Les substitutions dans le gène NF1 sont souvent retrouvées dans les répétitions d'homonucléotides ou dans les dinucléotides CpG. Les dinucléotides CpG méthylés ont souvent un haut taux de mutation du fait de la tendance de la 5-méthylcytosine à subir une désamination spontanée en thymine [19]. La plus fréquente des mutations $N F 1$ rapportée est une transition impliquant un site CpG dans l'exon 31 (R1947X) qui représente environ 1 à $2 \%$ des mutations identifiées. Néanmoins, seule une petite fraction des mutations $N F 1$ surviennent sur des sites CpG [20].

Des gènes ayant une certaine similarité de séquence avec $N F 1$ sont présents sur les chromosomes 2, 12, 14, $15,18,20,21$ et $22[21,22]$. La conversion génique entre un pseudogène non homologue et $N F 1$ pourrait produire quelques mutations. Toutefois, la comparaison des séquences mutées et des pseudo-gènes connus n'est pas en faveur de cette hypothèse.

Il est possible que la mutation $N F 1$ dans une cellule germinale donne un avantage prolifératif à cette cellule, ce qui donnerait lieu à un mosaïcisme germinal augmentant le risque de transmission. Un mosaïcisme germinal a été démontré chez un individu sain ayant donné naissance à deux enfants atteints [23]. Il pourrait expliquer pourquoi plusieurs autres familles ont plus d'un enfant atteint né de parents apparemment sains. Ce phénomène est cependant très rare.

Plus de $80 \%$ des néo-mutations NF1 sont d'origine paternelle [19]. Les "grandes" délétions sub-microscopiques de l'ensemble ou d'une grande partie du gène $N F 1$, qui représentent $5 \%$ de toutes les mutations, font exception à la règle car la plupart d'entre elles sont d'origine maternelle [24, 25].

Compte tenu de la prédominance des mutations d'origine paternelle, l'âge paternel a été proposé comme 
facteur de risque. Les données actuelles permettent de conclure que, s'il existe un effet, celui est modeste.

Les différentes mutations constitutionnelles de NF1 sont disponibles sur le site de la banque de données du Consortium d'Analyse Génétique de la NF1 (http://www.nf.org/ nflgene/). Le Tableau I résume les différents types de mutations détectées. La majorité d'entre elles donnent lieu à une neurofibromine tronquée; seuls environ $10 \%$ impliquent des substitutions d'acides aminés et moins de $2 \%$ concernent la région 3 ' non codante. Elles ont été identifiées tout au long du gène $N F 1$, sans identification d'un réel point chaud. Néanmoins, le type de mutations décrites dépend énormément de la technique utilisée pour les rechercher. Cela peut conduire à une surreprésentation des mutations faciles à détecter (par exemple les grandes délétions) ou au contraire à une sous-estimation des mutations plus difficiles à identifier (par exemple les mutations de la région 3' non codante). Aucune des méthodes utilisées ne permet d'identifier tous les types de mutations. Néanmoins, l'utilisation des différentes techniques disponibles permet l'identification de $95 \%$ d'entre elles [26].

\section{Procréation, mortalité, morbidité}

La fertilité des malades atteints de NF1 a été évaluée à environ 0,5 , c'està-dire à la moitié de la fertilité normale; elle serait plus altérée chez les hommes que chez les femmes [11]. Cette diminution de la fertilité n'étant pas liée aux avortements spontanés, elle est actuellement attribuée à la difficulté rencontrée par les malades à fonder un couple, difficulté plus marquée chez les hommes. Les autres causes avancées sont la morbidité et la mortalité associées à la NF1. La NF1 est une maladie évolutive, imprévisible, avec de nombreuses manifestations et complications associées à une diminution de l'espérance de vie. Néanmoins, la plupart des décès surviennent après l'âge habituel de la procréation.

La survie des malades atteints de NF1 est significativement diminuée par rapport à celle de la population générale [27, 28]. Les causes les plus communes de décès, cancer, infarctus du myocarde, accidents vasculaires cérébraux et pneumopathies, sont identiques à celles de la population générale, mais surviennent plus tôt dans la vie. Les tumeurs du système nerveux central, en particulier les gliomes, et les autres néoplasies sont plus fréquentes chez ces malades. L'âge moyen de décès est de l'ordre de 61 ans pour une espérance de vie attendue de 75 ans.

\section{Expression clinique de la NF1 et bases moléculaires de sa variabilité}

\section{Expression clinique} de la neurofibromatose 1

La NF1 est caractérisée par la présence de taches café au lait, de neurofibromes et de nodules de Lisch, et par une prédisposition aux difficultés d'apprentissage, aux dysplasies congénitales et aux cancers [29, 30]. Le diagnostic de NF1 est établi chez un individu si deux ou plus des 7 critères suivants sont rencontrés [12]: (1) au moins six taches café au lait de plus de $5 \mathrm{~mm}$ dans leur plus grand

Tableau I. Résumé des différents types de mutations NF1.

\begin{tabular}{|lcc|}
\hline Mutations & Nombre & $\%$ \\
\hline Anomalies chromosomiques & 4 & 1,6 \\
Délétion du gène entier & 18 & 7,3 \\
Délétions multiples d'exons & 38 & 15,4 \\
Petite délétion & 55 & 22,4 \\
Grande délétion & 3 & 1,2 \\
Petite insertion & 27 & 11 \\
Mutation stop & 43 & 17,5 \\
Substitution d'acides aminés & 29 & 11,8 \\
Mutation intronique & 25 & 10,2 \\
Mutation non codante de la région 3' & 4 & 1,6 \\
Total & $\mathbf{2 4 6}$ & \\
\hline
\end{tabular}

diamètre chez des individus prépubères et de plus de $15 \mathrm{~mm}$ chez des individus pubères (figure 1) ; (2) deux neurofibromes (figure 2) ou plus de n'importe quel type ou un neurofibrome plexiforme (figure 3); (3) des "éphélides» axillaires (figure 4) ou inguinales; (4) un gliome des voies optiques (figure 5); (5) deux nodules de Lisch ou plus (hamartomes iriens) (figure 6); (6) une lésion osseuse caractéristique (figures 7,8 , et 9) comme une dysplasie sphénoïde, un amincissement de la corticale des os longs avec ou sans pseudarthrose; (7) un parent du premier degré atteint de NF1 suivant les critères précédents.

Les complications tumorales de la NF1 sont causes de morbidité et de mortalité au cours de la NF1: neurofibromes plexiformes, tumeurs malignes des gaines nerveuses, et tumeurs du système nerveux central. Les neurofibromes plexiformes [31, 32] sont l'une des plus fréquentes causes de morbidité et d'altérations esthétiques liées à la NF1; environ $30 \%$ des malades en sont atteints. Il s'agit de proliférations cellulaires de la gaine des nerfs impliquant plusieurs faisceaux nerveux, qui peuvent être visibles ou se développer en profondeur. Ils peuvent se développer à partir des paires de nerfs crâniens, la plus fréquemment atteinte étant le nerf trijumeau. Ces neurofibromes ont tendance à pousser dans l'orbite, causant une proptose, et sont souvent associés à une dysplasie de la grande aile du sphénoïde. Les neurofibromes plexiformes impliquant le tronc et les membres peuvent s'associer à une augmentation de la croissance pouvant conduire à une hypertrophie significative. Dans certains cas, les neurofibromes plexiformes se développent à partir des plexus brachiaux ou sacrés, et s'étendent aux nerfs spinaux et aux gros troncs nerveux des membres. Ils peuvent également atteindre le tractus digestif et la sphère génito-urinaire.

La croissance des neurofibromes peut survenir tout au long de la vie. L'expérience clinique suggère néanmoins que cette croissance se produit essentiellement au cours de deux périodes : - la prime enfance, la croissance étant alors rapide durant plusieurs années; - au moment des modifications hormonales, notam- 


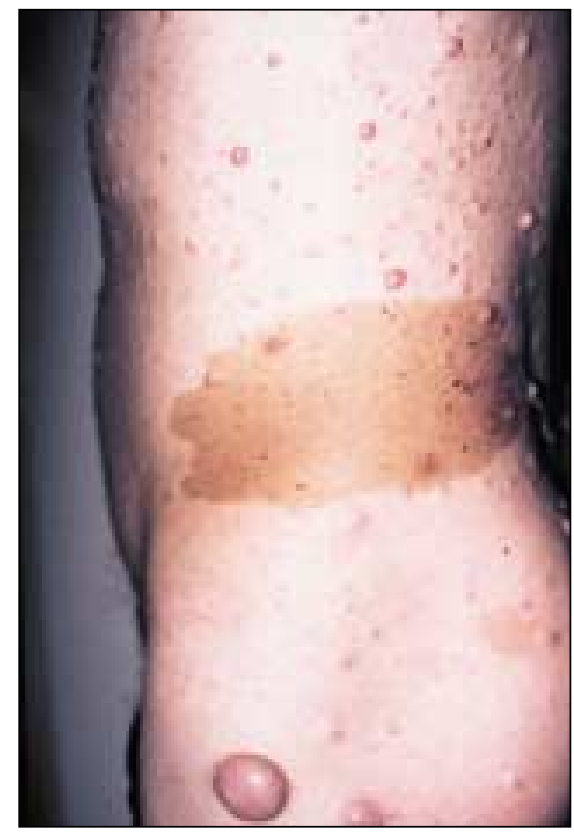

Figure 1. Taches café au lait.

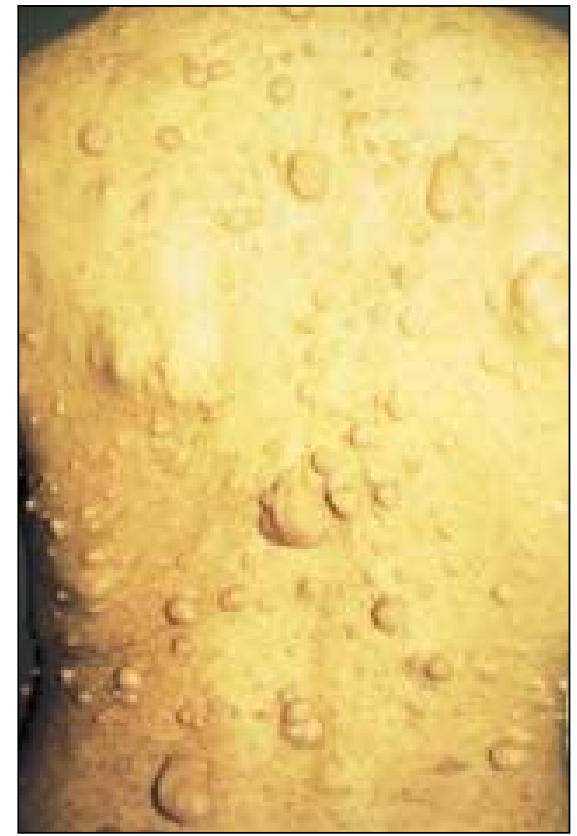

Figure 2. Neurofibromes cutanés multiples.

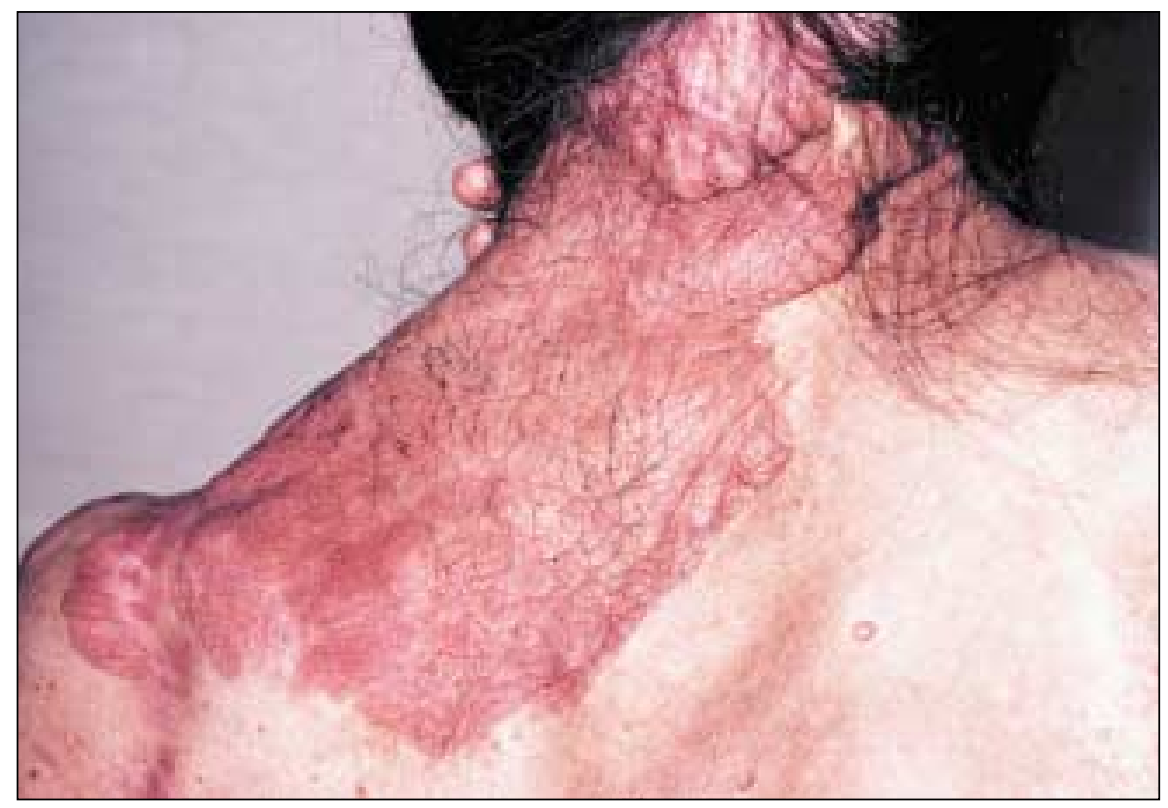

Figure 3. Neurofibrome plexiforme.

ment durant la puberté et chez les femmes au cours de la grossesse, conduisant alors parfois à l'envahissement ou à la compression des organes voisins. Même si toutes les tumeurs malignes des gaines nerveuses ne surviennent pas sur un neurofibrome plexiforme préexistant, il est clair que ces tumeurs sont à risque accru de dégénérescence maligne [33, 34]. les petits neurofibromes plexiformes. Les autres types de traitement, radiothérapie et chimiothérapie, n'ont pas été évalués. Il n'est pas évident que ces traitements puissent avoir une action sur des tumeurs à croissance lente, et ils pourraient provoquer des néoplasies secondaires.

Les gliomes des voies optiques [3537] ne représentent que 2 à $5 \%$ de toutes les tumeurs cérébrales, mais $70 \%$ d'entre eux sont associés à la NF1. Ces tumeurs touchent essentiellement les voies visuelles antérieures: nerf optique intra-orbitaire, nerf optique intra-crânien, et chiasma optique. Histologiquement, il s'agit d'astrocytomes pilocytiques dont l'incidence oscille, suivant les études, entre $1,5 \%$ et $7,5 \%$ des malades atteints de NF1. Ils surviennent principalement dans l'enfance durant les 6 premières années de la vie et évoluent généralement de manière bénigne mais, chez certains individus, peuvent conduire à un déficit visuel, à une puberté précoce, voire $\mathrm{au}$ décès. Ces tumeurs évoluent de deux manières: (1) la moitié ne causent aucun symptôme ou manifestation, et seule la détection systématique par l'imagerie les découvrira; (2) l'autre moitié provoque des symptômes visuels ou des signes physiques (proptose, diminution de l'acuité visuelle et puberté précoce). Les deux tiers des tumeurs des voies optiques touchent le chiasma. Le symptôme le plus impressionnant est l'apparition rapide, chez environ $30 \%$ des malades présentant une tumeur symptomatique, d'une proptose s'accompagnant de troubles de la vision. Dans un tiers des cas, le premier signe de gliome des voies optiques est la puberté précoce.

En cas d'évolution agressive d'un gliome des voies optiques, trois types de traitements ont été proposés, la chirurgie, la radiothérapie et la chimiothérapie. La chirurgie a un intérêt limité. En effet dans les gliomes du nerf optique, elle ne prévient pas la dissémination tumorale au chiasma et au nerf controlatéral; dans les gliomes du chiasma, elle peut aggraver la perte visuelle et la morbidité neurologique. La radiothérapie permet de stopper au moins transitoirement l'évolution tumorale ; elle a donc longtemps été considérée comme la thérapeutique de choix. 

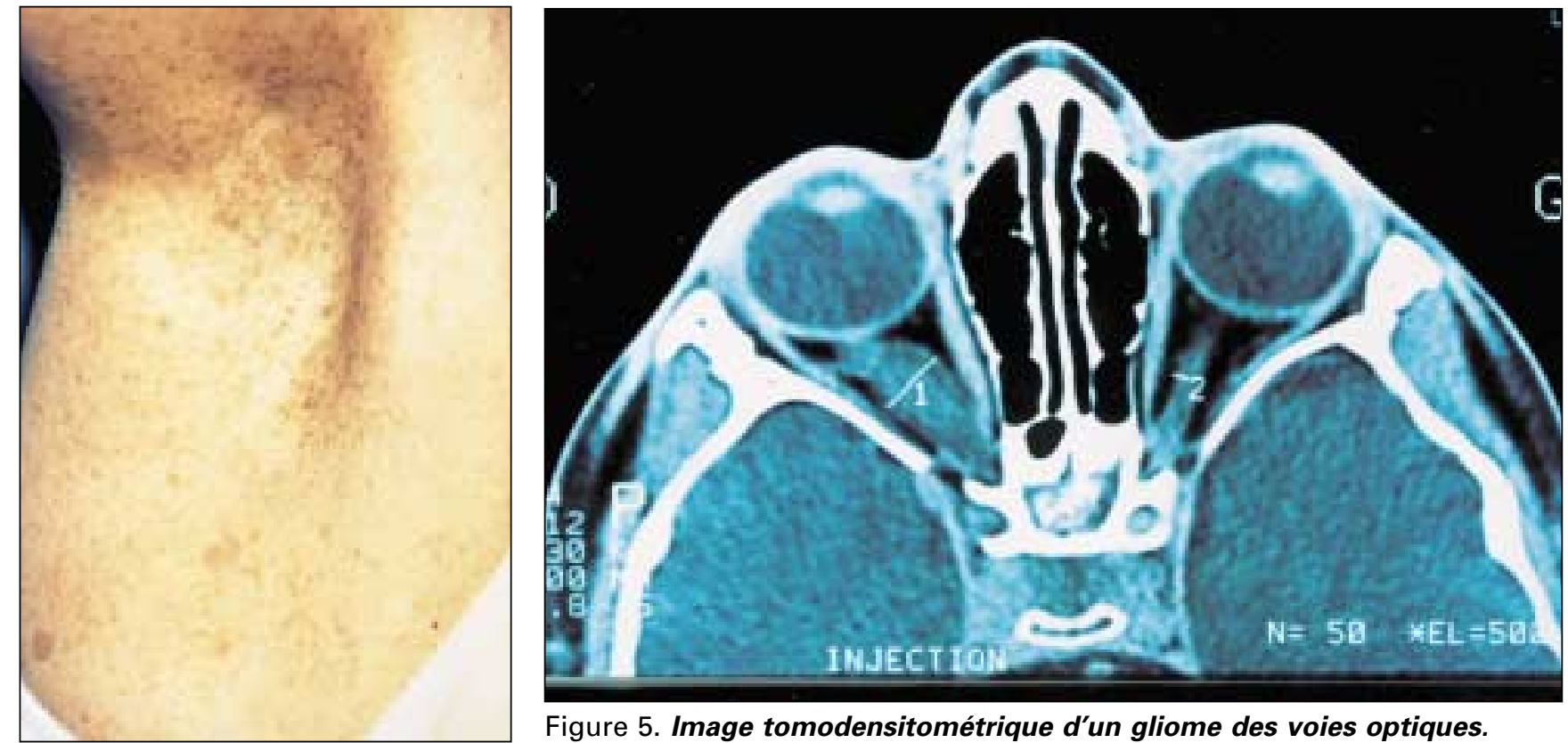

Figure 5. Image tomodensitométrique d'un gliome des voies optiques.

Figure 4. Éphélides axillaires.

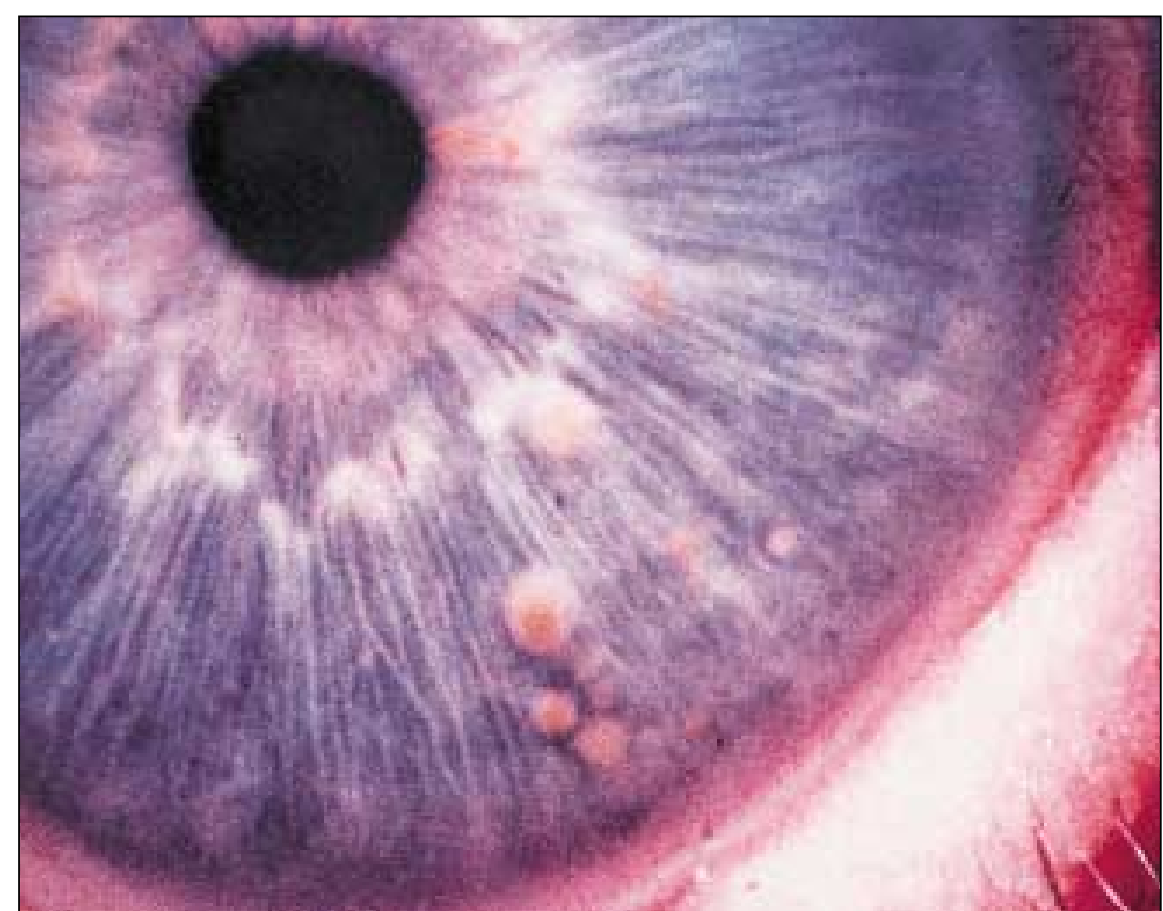

Figure 6. Nodules de Lisch.

Néanmoins, la radiothérapie a des effets secondaires endocrinologiques et neurocognitifs bien connus et présente un risque à long terme de vasculopathie occlusive; de plus, la survie à 20 ans des malades semble équivalente qu'ils aient eu ou non résultats médiocres et des effets secondaires, un regain d'intérêt récent a eu lieu pour l'utilisation de chimiothérapies par carboplatine seul ou combiné à de la vincristine. En effet, l'association carboplatine et vincristine permet d'obtenir une réduction tumorale avec améliora-

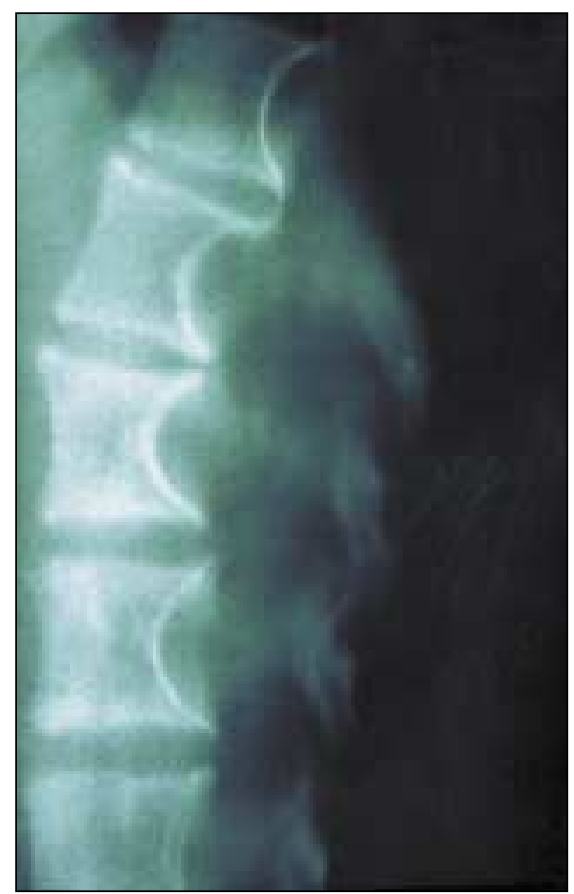

Figure 7. Dystrophie osseuse caractéristique de la neurofibromatose 1 : scalopping vertébral.

tion clinique et un accroissement de la survie sans progression [38]. Ces résultats sont très encourageants. Deux stratégies thérapeutiques peuvent être, en effet, envisagées dans le futur. La première conduirait à utiliser la chimiothérapie comme un moyen de freiner la croissance tumo- 


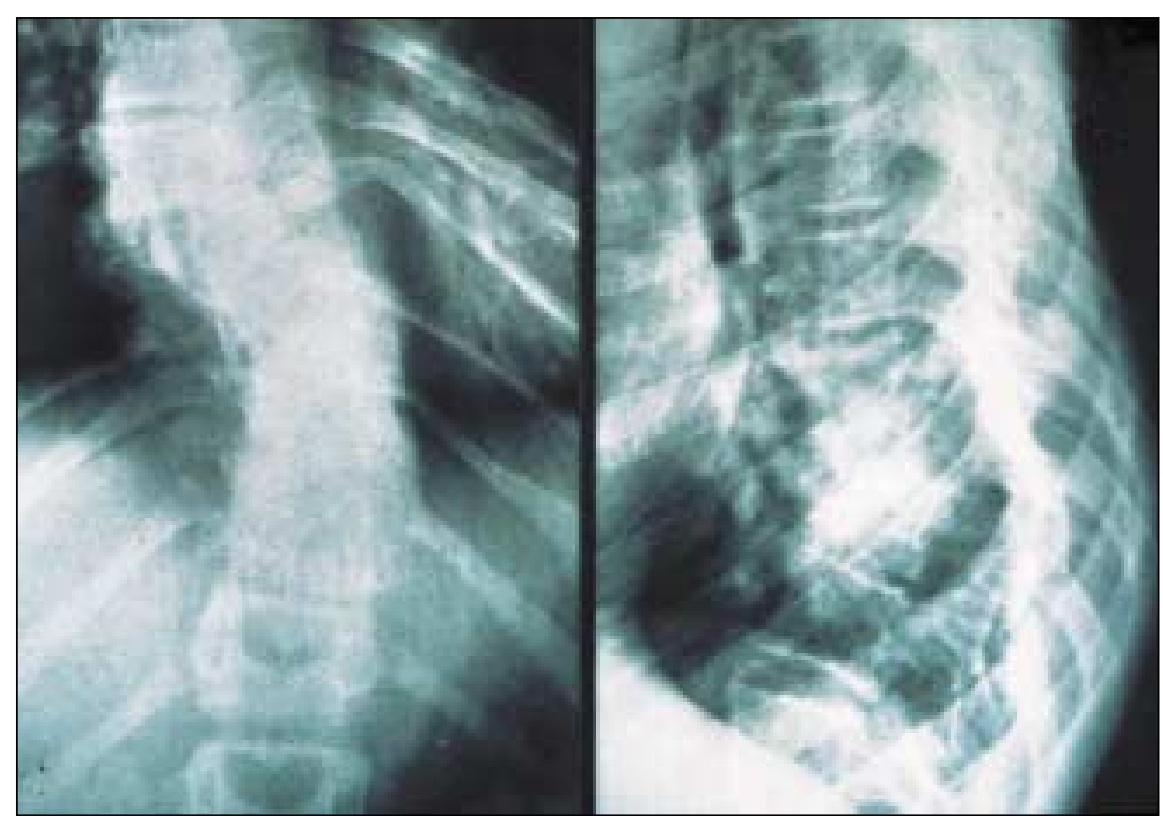

Figure 8. Scoliose thoracique.

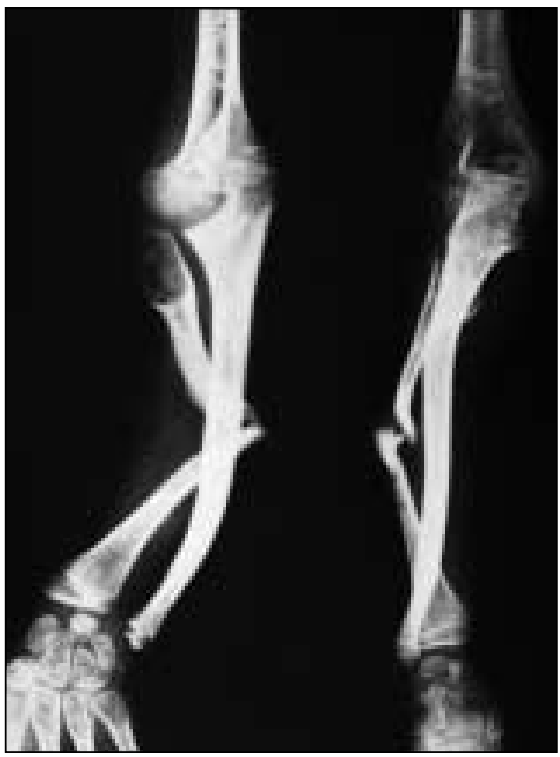

Figure 9. Pseudarthrose tibiale.

rale jusqu'à ce que les enfants malades atteignent l'âge suffisant pour être traités par irradiation sans effet secondaire majeur. La deuxième conduirait à utiliser ces chimiothérapies durant la période de croissance tumorale majeure (avant l'âge de 6 ans) et permettraient peutêtre de se passer de tout traitement ultérieur. Des études complémentaires et un suivi à long terme sont nécessaires pour évaluer cette alternative.

Les autres gliomes cérébraux [37,
39] associés à la NF1 sont encore mal connus. Le principal obstacle est la confusion faite avec les "objets brillants non identifiés» (OBNI), imagerie par résonance magnétique dans le cerveau d'environ $60 \%$ des malades. Même si les caractéristiques histologiques de ces OBNI ne sont pas bien connues, ils ont une évolution clinique bénigne et disparaissent habituellement avec l'âge. En fait, ces OBNI sont facilement distingués des gliomes car ils ne sont pas rehaussés par le produit de contraste et n'induisent pas d'effet de masse. Comme pour le gliome des voies optiques, le pronostic des gliomes intra-cérébraux apparaît bien meilleur chez les malades NF1 que dans la population générale.

\section{Bases moléculaires de la variabilité d'expression clinique}

La variabilité de l'expression clinique au cours de la NF1 est une caractéristique de la maladie, et elle est à la fois intra- et interfamiliale [40]. Au sein de la même famille, les individus ayant hérité d'une même mutation $N F 1$, les malades présentent des différences phénotypiques marquées allant d'une forme bénigne à une forme grave. Une liste de mécanismes a été proposée pour expliquer cette grande variabilité: l'hétérogénéité hypersignaux en T2 retrouvés en allélique, la deuxième inactivation de ce gène suppresseur de tumeur, le mosaïcisme somatique, la délétion de gènes contigus, l'intervention de gènes modificateurs, les facteurs épigénétiques, les facteurs environnementaux... et le hasard [29].

L'hétérogénéité allélique a permis d'expliquer certaines formes très particulières de NF1, comme la neurofibromatose spinale, la neurofibromatose gastro-intestinale ou les taches café au lait familiales. Une autre explication de la variabilité des manifestations cliniques de la NF1 est l'inactivation somatique de l'allèle NF1 normal. Ce concept bien établi est relié à la notion du rôle de suppresseur de tumeur accordé à $N F 1$. Ainsi, la perte d'hétérozygotie et l'inactivation de l'autre allèle $N F 1$ ont été établies dans un certain nombre de néoplasies associées à NF1, incluant des neurofibromes cutanés, des tumeurs malignes des gaines nerveuses, des phéochromocytomes et des dysplasies myéloïdes [41-43]. Des études récentes ont permis de préciser que l'inactivation du gène NF1 dans les cellules de Schwann, et non pas dans les fibroblastes, était à l'origine des neurofibromes [44-46].

Cependant, l'inactivation de l'allèle normal ne permet pas d'expliquer de nombreuses manifestations de la NF1, notamment les difficultés d'apprentissage et les dysplasies osseuses. Un mosaïcisme somatique a été établi chez certains malades [23]. Ce mosaïcisme peut conduire dans certains cas à des formes à début tardif ou à des phénotypes atténués.

Une autre explication serait l'intervention de gènes modificateurs [40]. Quatre traits de la NF1, épilepsie, gliomes des voies optiques, difficultés d'apprentissage et scoliose, semblent avoir un regroupement familial significatif, démontrant la ségrégation de gènes épistatiques non liés au locus NF1. Les pseudarthroses tibiales et les syndromes myélodysplasiques semblent d'autre part prédominer chez les individus de sexe masculin et le gliome des voies optiques être beaucoup moins fréquent chez les malades noirs américains que chez les autres. En ce qui concerne l'intervention de facteurs environnementaux, un rôle des traumatismes physiques a été suggéré; la prise de contraceptifs oraux et 
la grossesse ont également été suggérés mais les preuves manquent. La stochastique pourrait également modifier l'expression: tout clinicien ayant observé la distribution des taches café au lait et des neurofibromes évoquera effectivement le hasard.

La délétion de gènes contigus pourrait enfin modifier le phénotype. Ainsi un phénotype particulier associant apparition précoce de nombreux neurofibromes, dysmorphie et retard mental paraît lié à de grandes délétions du gène $N F 1$ [47].

\section{Hyperactivité de Ras au cours de la NF1}

\section{Rôle de Ras}

Les protéines Ras jouent un rôle central dans la croissance et la différen-

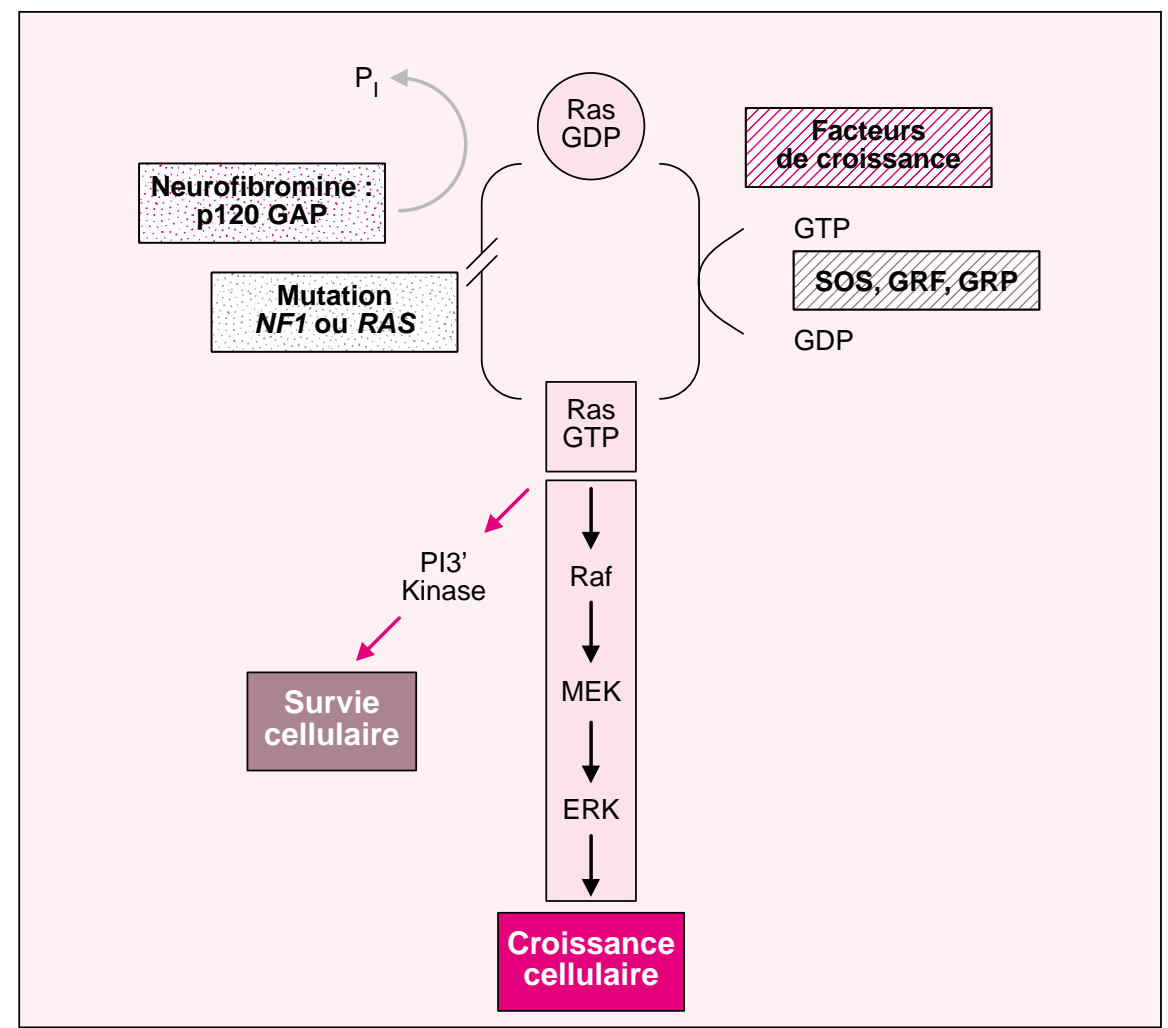

Figure 10. Schématisation de l'activation de Ras. La production de Ras est finement réglée par un cycle entre une conformation active porteuse de GTP et une conformation inactive porteuse de GDP. Ras a une activité intrinsèque GTPasique lente, augmentée par GAP. Les protéines Ras contrôlent la mort cellulaire en conduisant un signal de la membrane jusqu'au noyau au travers d'une série d'effecteurs en cascade. Ras GTP recrute la Raf kinase à la membrane où son activité kinase est effective. Raf, en retour, active une cascade de kinases impliquant la MEK et les isoformes Erk1 et Erk2 de la MAP kinase. L'état d'activation de la phosphoinositol-3'-kinase (PI3K) est aussi réglé par Ras GTP dans de nombreux types cellulaires. La neurofibromine possède un teurs en cascade [50]. Ras GTP recrute la Raf kinase à la membrane où son activité kinase est effective. Raf, en retour, active une cascade de kinases impliquant la MEK kinase et les isoformes Erk1 et Erk2 de la MAP kinase. L'état d'activation de la phosphoinositol-3'-kinase (PI3K) et les voies Rac/Rho sont aussi règlés par Ras GTP dans de nombreux types cellulaires. Les conséquences de l'activation de Ras sont influencées par le contexte cellulaire et par l'influence réciproque entre les différentes cascades effectrices.

La neurofibromine possède un domaine comparable à GAP (GAPrelated domain - GRD) et l'analyse biochimique de peptides recombinants correspondant à GRD a permis de démontrer que la protéine est une authentique GAP pour Ras [51]. L'activité GAP de la neurofibromine a de profondes implications à la fois dans la compréhension des complications de la NF1, et pour la conception rationnelle de thérapeutiques $[52,53]$. En effet des agents thérapeutiques ciblés sur les voies effectrices de Ras comme les inhibiteurs de MEK ou de PI3K peuvent éventuellement être envisagés dans le futur.

\section{Hyperactivité de Ras dans les complications tumorales de la NF1}

Les cellules dérivées de malades atteints de NF1 et de souris dont le gène $N f 1$ a été invalidé ont été utilisées pour tester l'hypothèse selon laquelle $N F 1$ fonctionnerait comme un gène suppresseur de tumeur, et pour déterminer si sa perte de fonction est associée à une hyperactivité de Ras [54, 55]. Les sujets atteints de NF1 sont prédisposés à des cancers spécifiques, les tumeurs malignes des gaines nerveuses [56], les astrocytomes, les phéochromocytomes et la leucémie myélomonocytaire juvénile [42, 43, 57]. Une perte d'hétérozygotie somatique a été démontrée au cours de chacun de ces cancers associés à la NF1. De manière similaire, les souris hétérozygotes pour l'invalidation du gène $N f 1$ sont prédisposées à ces néoplasies, et la perte de l'allèle sauvage $N f 1$ y est démontrée [14, 15]. Un certain nombre de données suggèrent que l'inactivation de $N F 1$ est 
associée à une hyperactivité de Ras dans les cellules tumorales. Les lignées de cellules de tumeurs malignes des gaines nerveuses ont une activité GAP diminuée in vitro, et un niveau élevé de Ras GTP, retrouvé dans les tumeurs des malades et dans les cellules de Schwann isolées d'embryons de souris homozygotes pour l'invalidation du gène NfI.

Le rôle de la neurofibromine dans la croissance des cellules myéloïdes a été également étudié chez l'homme et la souris. Les cellules leucémiques issues d'enfants atteints ont une activité GAP-neurofibromine réduite, des niveaux élevés de Ras GTP et une activation de MAP kinase. Les cellules hématopoïétiques d'embryons de souris $\mathrm{NfI}^{-/-}$ont une activité constitutive de MAP kinase et une hyperactivité de la réponse aux facteurs de croissance hématopoïétiques.

Ces données humaines et murines laissent supposer que la fonction suppresseur de tumeur de NF1 est liée à la capacité de la neurofibromine à régler négativement Ras. Ainsi l'expression des domaines GAP de la neurofibromine permet-elle de restaurer la croissance normale des cellules $N f 1^{-/-}[58]$. De plus, même si la plupart des mutations NF1 donnent lieu à une neurofibromine tronquée et sont donc censées donner un allèle nul, quelques familles avec des mutations faux-sens ont été rapportées. Dans un cas, une mutation fauxsens avec conservation du domaine GAP n'était pas associée avec un phénotype particulier. Une autre famille avec une autre mutation faux-sens a été récemment décrite. Cette mutation n'influence pas la structure secondaire et tertiaire de la protéine, ne réduit pas le niveau de la neurofibromine, et n'influence pas de manière significative la liaison avec Ras. En revanche, l'analyse structurale a démontré un effondrement de l'activité GAP, et les phénotypes observés étaient typiques de NF1. Cet exemple suggère que les phénotypes cliniques associés à la NF1 peuvent être expliqués par une capacité sélective de la neurofibromine à interagir et à régler négativement Ras [59].

L'inactivation d'un seul allèle $N F 1$ pourrait induire des altérations biochimiques subtiles pouvant expliquer les difficultés d'apprentissage et les anomalies de pigmentation. La souris hétérozygote $N f 1$ a des difficultés d'apprentissage spécifiques compatibles avec cette hypothèse [60]. Le rôle du domaine GAP de NF1 comme modulateur de l'apprentissage et de la mémoire a d'ailleurs été récemment démontré [61].

\section{Conclusions : perspectives thérapeutiques}

L'activité biologique de Ras nécessite sa modification post-traductionnelle par le tranfert de groupes isoprénoïdes donnés par un geranylgeranyl pyrophosphate et un farnasyl pyrophosphate. Un certain nombre d'inhibiteurs de la farnesyl transférase, dont l'activité antitumorale était manifeste lors d'études sur des systèmes in vivo et in vitro, sont actuellement expérimentés dans des protocoles de phase 1 ou 2 chez des malades ayant des cancers réfractaires aux thérapeutiques conventionnelles. L'idée d'un Ras hyperactif contribuant à la prolifération cellulaire anormale donne un rationnel à l'utilisation de ces composés. Une deuxième perspective thérapeutique pour les complications de la NF1 est sous-tendue par le fait que les cellules déficientes en NF1 sont hypersensibles à certains facteurs de croissance. Des antagonistes de ces facteurs de croissance représenteraient une approche thérapeutique rationnelle. Des essais sont par ailleurs actuellement en cours avec des thérapeutiques anti-prolifératives, comme l'interféron-alpha, ou anti-angiogènes, comme le thalidomide.

Les stratégies de thérapie génique sont une autre voie de recherche séduisante, mais la correction des mutations NF1 dans les tissus atteints se heurte à des obstacles majeurs. La grande taille du gène rend difficile la construction de vecteurs qui pourraient transduire et exprimer efficacement la neurofibromine. L'utilisation d'un plus petit fragment d'ADN codant pour le domaine GAP serait une solution potentielle à ce problème ; cependant, il n'est pas certain qu'il serait correctement contrôlé ou qu'il fonctionnerait comme une neurofibromine entière. Enfin, un nombre élevé de cellules doit être corrigé dans différents tissus, et la neurofibromine devrait être produite à des taux physiologiques

\section{RÉFÉRENCES}

1. Huson SM. The neurofibromatoses : a pathogenetic and clinical overview, 1st ed. London: Chapmann and Hall, 1994.

2. Viskochil D. Neurofibromatosis 1. Introduction. Am J Med Genet 1999; 89 : v-viii.

3. Barker D, Wright E, Nguyen K, et al. Gene for von Recklinghausen neurofibromatosis is in the pericentromeric region of chromosome 17. Science 1987; 236: 1100-2.

4. Marchuk DA, Saulino AM, Tavakkol R, et al. cDNA cloning of the type 1 neurofibromatosis gene: complete sequence of the NF1 gene product. Genomics 1991 ; 11: 931-40.

5. Gutmann DH, Wood DL, Collins FS. Identification of the neurofibromatosis type 1 gene product. Proc Natl Acad Sci USA 1991; 88: 9658-62.

6. Ballester R, Marchuk D, Boguski M, et al. The NF1 locus encodes a protein functionally related to mammalian GAP and yeast IRA proteins. Cell 1990; 63: 851-9.

7. Weiss B, Bollag G, Shannon K. Hyperactive Ras as a therapeutic target in neurofibromatosis type 1. Am I Med Genet 1999; 89 : 14-22.

8. Nordlund M, Gu X, Shipley MT, Ratner $\mathrm{N}$. Neurofibromin is enriched in the endoplasmic reticulum of CNS neurons. $J \mathrm{Neu}$ rosci 1993; 13 : 1588-600.

9. Upadhyaya M, Shaw DJ, Harper PS. Molecular basis of neurofibromatosis type 1 (NF1) : mutation analysis and polymorphisms in the NF1 gene. Hum Mutat 1994; 4 : 83-101.

10. Friedman JM Epidemiology of neurofibromatosis type 1. Am J Med Genet 1999; 89 : 1-6.

11. Huson SM, Compston DA, Clark P, Harper PS. A genetic study of von Recklinghausen neurofibromatosis in south east Wales. I. Prevalence, fitness, mutation rate, and effect of parental transmission on severity. $J$ Med Genet 1989; 26 : 704-11.

12. Gutmann DH, Aylsworth A, Carey JC, et al. The diagnostic evaluation and multidisciplinary management of neurofibromatosis 1 and neurofibromatosis 2. JAMA 1997; 278 : 51-7.

13. Rasmussen SA, Friedman JM. NF1 gene and neurofibromatosis 1. Am J Epidemiol 2000; $151: 33-40$.

14. Brannan CI, Perkins AS, Vogel KS, et al. Targeted disruption of the neurofibromatosis type-1 gene leads to developmental abnormalities in heart and various neural crest-derived tissues. Genes Dev 1994; 8 : 1019-29.

15. Jacks T, Shih TS, Schmitt EM, et al. Tumour predisposition in mice heterozygous for a targeted mutation in Nf1. Nat Genet 1994; 7 : 353-61. 


\section{RÉFÉRENCES}

16. Shen MH, Harper PS, Upadhyaya M. Molecular genetics of neurofibromatosis type 1 (NF1). J Med Genet 1996; 33: 2-17.

17. Upadhyaya M, Shaw DJ, Harper PS Molecular basis of neurofibromatosis type 1 (NF1): mutation analysis and polymorphisms in the NF1 gene. Hum Mutat 1994; 4 : 83-101.

18. Gasparini P, Grifa A, Origone P, et al. Detection of a neurofibromatosis type I (NF1) homologous sequence by PCR : implications for the diagnosis and screening of genetic diseases. Mol Cell Probes 1993; 7: $415-8$.

19. Lazaro C, Gaona A, Ainsworth P, et al. Sex differences in mutational rate and mutational mechanism in the NF1 gene in neurofibromatosis type 1 patients. Hum Genet 1996; 98: 696-9.

20. Boulandet EG, Pantel J, Cazeneuve C, et al. NF1 gene analysis focused on CpG-rich exons in a cohort of 93 patients with neurofibromatosis type 1. Hum Mutat 2000; 16 : 274-5.

21. Purandare SM, Huntsman BH, Li Y, et al. Identification of neurofibromatosis 1 (NF1) homologous loci by direct sequencing, fluorescence in situ hybridization, and PCR amplification of somatic cell hybrids. Genomics 1995; 30 : 476-85.

22. Ritchie RJ, Mattei MG, Lalande M. A large polymorphic repeat in the pericentromeric region of human chromosome $15 q$ contains three partial gene duplications. Hum Mol Genet 1998; 7 : 1253-60.

23. Lazaro C, Ravella A, Gaona A, et al. Neurofibromatosis type 1 due to germ-line mosaicism in a clinically normal father [see comments]. N Engl J Med 1994; 331 : 1403-7.

24. Upadhyaya M, Ruggieri M, Maynard J, et al. Gross deletions of the neurofibromatosis type 1 (NF1) gene are predominantly of maternal origin and commonly associated with a learning disability, dysmorphic features and developmental delay. Hum Genet 1998; 102: 591-7.

25. Correa CL, Brems H, Lazaro C, et al. Unequal meiotic crossover: a frequent cause of NF1 microdeletions. Am J Hum Genet 2000; 66 : 1969-74.

26. Messiaen LM, Callens T, Mortier G, et al. Exhaustive mutation analysis of the NF1 gene allows identification of $95 \%$ of mutations and reveals a high frequency of unusual splicing defects. Hum Mutat 2000; 15 : 541-55.

27. Sorensen SA, Mulvihill IJ, Nielsen A. Long-term follow-up of von Recklinghausen neurofibromatosis. Survival and malignant neoplasms. N Engl J Med 1986; 314: 1010-5.

28. Zoller M, Rembeck B, Akesson HO, Angervall L. Life expectancy, mortality and type 1. A twelve-year follow-up of an epidemiological study in Goteborg, Sweden. Acta Dermatol Venereol 1995; 75 : 136-40.

29. Carey JC, Viskochil DH. Neurofibromatosis type 1: A model condition for the study of the molecular basis of variable expressivity in human disorders. $\mathrm{Am} \mathrm{J} \mathrm{Med}$ Genet 1999; 89: 7-13.

30. Friedman JM, Birch PH. Type 1 neurofibromatosis: a descriptive analysis of the disorder in 1,728 patients. Am J Med Genet 1997 ; 70 : 138-43.

31. Korf BR. Plexiform neurofibromas. Am J Med Genet 1999; 89 : 31-7.

32. Waggoner DI, Towbin J, Gottesman G, Gutmann DH. Clinic-based study of plexiform neurofibromas in neurofibromatosis 1. Am J Med Genet 2000; 92 : 132-5.

33. King AA, Debaun MR, Riccardi VM, Gutmann DH. Malignant peripheral nerve sheath tumors in neurofibromatosis 1 . Am J Med Genet 2000; 93: 388-92.

34. Woodruff JM. Pathology of tumors of the peripheral nerve sheath in type 1 neurofibromatosis. Am J Med Genet 1999; 89: 2330.

35. Listernick R, Charrow J, Greenwald M, Mets M. Natural history of optic pathway tumors in children with neurofibromatosis type 1: a longitudinal study. J Pediatr 1994; $125: 63-6$.

36. Listernick R, Louis DN, Packer RJ, Gutmann DH. Optic pathway gliomas in children with neurofibromatosis 1: consensus statement from the NF1 optic pathway glioma task force. Ann Neurol 1997; 41 : 1439 .

37. Listernick R, Charrow J, Gutmann DH. Intracranial gliomas in neurofibromatosis type 1. Am J Med Genet 1999; 89: 38-44.

38. Listernick R, Charrow J, Tomita T, Goldman S. Carboplatin therapy for optic pathway tumors in children with neurofibromatosis type-1. J Neurooncol 1999; 45 : 185-90.

39. Creange A, Zeller J, Rostaing-Rigattieri $\mathrm{S}$, et al. Neurological complications of neurofibromatosis type 1 in adulthood. Brain 1999; 122: 473-81.

40. Easton DF, Ponder MA, Huson SM, Ponder BA. An analysis of variation in expression of neurofibromatosis (NF) type 1 (NF1) : evidence for modifying genes. Am J Hum Genet 1993; 53 : 305-13.

41. Sawada S, Florell S, Purandare SM, et al. Identification of NF1 mutations in both alleles of a dermal neurofibroma. Nat Genet $1996 ; 14: 110-2$.

42. Shannon KM, O'Connell P, Martin GA, et al. Loss of the normal NF1 allele from the bone marrow of children with type 1 neurofibromatosis and malignant myeloid disorders. N Engl J Med 1994; 330 : 597-601.
43. Xu W, Mulligan LM, Ponder MA, et al. Loss of NF1 alleles in phaeochromocytomas from patients with type I neurofibromatosis. Genes Chromosomes Cancer 1992; 4 : 337-42.

44. Kluwe L, Friedrich R, Mautner VF. Loss of NF1 allele in Schwann cells but not in fibroblasts derived from an NF1-associated neurofibroma. Genes Chromosomes Cancer $1999 ; 24: 283-5$.

45. Serra E, Rosenbaum T, Winner U, et al. Schwann cells harbor the somatic NF1 mutation in neurofibromas: evidence of two different Schwann cell subpopulations. Hum Mol Genet 2000; 9 : 3055-64.

46. Sherman LS, Atit R, Rosenbaum T, et al. Single cell Ras-GTP analysis reveals altered Ras activity in a subpopulation of neurofibroma Schwann cells but not fibroblasts. I Biol Chem 2000; 275 : 30740-5.

47. Rasmussen SA, Colman SD, Ho VT, et al. Constitutional and mosaic large NF1 gene deletions in neurofibromatosis type $1 . \mathrm{J} \mathrm{Med}$ Genet 1998; 35 : 468-71.

48. Buchberg AM, Cleveland LS, Jenkins NA, Copeland NG. Sequence homology shared by neurofibromatosis type-1 gene and IRA-1 and IRA-2 negative regulators of the RAS cyclic AMP pathway. Nature 1990; 347: 291-4.

49. Xu GF, O'Connell P, Viskochil D, et al. The neurofibromatosis type 1 gene encodes a protein related to GAP. Cell 1990; 62 : 599608 .

50. Wittinghofer A. Signal transduction via Ras. Biol Chem 1998; 379: 933-7.

51. Bernards A Neurofibromatosis type 1 and Ras-mediated signaling: filling in the GAPs. Biochim Biophys Acta 1995; 1242: 4359.

52. Scheffzek K, Ahmadian MR, Kabsch W, et al. The Ras-RasGAP complex: structural basis for GTPase activation and its loss in oncogenic Ras mutants. Science 1997; 277 : 333-8.

53. Scheffzek K, Ahmadian MR, Wiesmuller L, et al. Structural analysis of the GAPrelated domain from neurofibromin and its implications. EMBO J 1998; 17: 431327

54. Basu TN, Gutmann DH, Fletcher JA, et al. Aberrant regulation of ras proteins in malignant tumour cells from type 1 neurofibromatosis patients. Nature 1992; 356: 713-5.

55. DeClue JE, Papageorge AG, Fletcher JA, et al. Abnormal regulation of mammalian p21ras contributes to malignant tumor growth in von Recklinghausen (type 1) neurofibromatosis. Cell 1992; 69: 265-73.

56. Legius E, Marchuk DA, Collins FS, Glover TW. Somatic deletion of the neurofibromatosis type 1 gene in a neurofibrosarcoma supports a tumour suppressor gene hypothesis. Nat Genet 1993; 3: 122-6. 


\section{RÉFÉRENCES}

57. Side L, Taylor B, Cayouette $\mathrm{M}$, et al. Homozygous inactivation of the NF1 gene in bone marrow cells from children with neurofibromatosis type 1 and malignant myeloid disorders. N Engl J Med 1997; 336 : 1713-20.

58. Hiatt KK, Ingram DA, Zhang Y, et al. Neurofibromin GTPase-activating proteinrelated domains restore normal growth in $\mathrm{Nf1}^{-1-}$ cells. J Biol Chem 2001; 276: 7240-5.

59. Klose A, Ahmadian MR, Schuelke M, et al. Selective disactivation of neurofibromin GAP activity in neurofibromatosis type 1 . Hum Mol Genet 1998; 7 : 1261-8

60. Silva AJ, Frankland PW, Marowitz Z, et $a l$. A mouse model for the learning and memory deficits associated with neurofibromatosis type I. Nat Genet 1997; 15 : 281-4

61. Costa RM, Yang T, Huynh DP, et al. Learning deficits, but normal development and tumor predisposition, in mice lacking exon 23a of Nf1. Nat Genet 2001; 27:399405 .

\section{Summary}

Neurofibromatosis 1

Neurofibromatosis 1 is an autosomal dominant disorder that affects about one subject out of 4000 . NF1 gene localized in $17 q 11.2$ is of about 335 kilobases and encodes a 2818 amino-acid protein, neurofibromin. It is a tumor-suppressor gene. Mutations have been identified along the whole gene without hotspots. In the same family in which all subjects have inherited of the same NF1 mutation, patients have different phenotypes, from benign to severe. Main complications of neurofibromatosis 1 are tumors that cause morbidity and mortality: plexiform neurofibromas, malignant peripheral nerve sheath tumors, central nervous system tumors. Inactivation of $N F 1$ in tumor cells seems to be associated with a hyperactive Ras. This opens new therapeutic prospects.

\section{FONDATION FYSSEN 194, rue de Rivoli - 75001 PARIS \\ Tél. : 33 (0) 142975316 - Fax: 33 (0) 142601795 http:///www.fondation-fyssen.org}

La FONDATION FYSSEN a pour objectif général «de promouvoir sous toutes ses formes l'analyse scientifique des mécanismes logiques du comportement animal et humain ainsi que leur développement ontogénétique et phylogénétique».

\section{BOURSES D'ÉTUDES POST-DOCTORALES}

La FONDATION FYSSEN attribuera un certain nombre de BOURSES D'ÉTUDES POSTDOCTORALES.

Ces bourses doivent permettre la formation et le soutien de chercheurs de niveau post-doctoral travaillant dans des domaines de recherche qui répondent aux objectifs de la Fondation tels que l'éthologie, la paléontologie, l'archéologie, l'anthropologie, la psychologie, l'épistémologie, la logique et les sciences du système nerveux.

La Fondation souhaiterait soutenir plus particulièrement les recherches dans les domaines tels que: ÉTHOLOGIE ET PSYCHOLOGIE : La nature et le développement des processus cognitifs chez l'homme et chez les animaux. Le déterminisme des comportements au cours de l'ontogenèse et leur évolution à travers la phylogenèse.

NEUROBIOLOGIE: Les études portant sur les bases neurobiologiques des processus cognitifs et de leur développement embryonnaire et post-natal ainsi que les mécanismes élémentaires qu'ils engagent.

ANTHROPOLOGIE-ETHNOLOGIE : L'étude

a) des systèmes de représentations des environnements naturels et des cultures. Analyse des principes de construction et des mécanismes de transmission de ces systèmes en mettant en évidence leurs aspects cognitifs ;

b) des systèmes techniques développés dans les diverses formes d'organisation sociale et analysés sous tous leurs aspects (savoirs, savoir-faire, mécanismes de transmission).

PALEONTOLOGIE HUMAINE - ARCHÉOLOGIE: L'origine et l'évolution du cerveau humain et des ses productions.

Ces bourses d'un montant maximum de $132000 \mathrm{~F}$ annuel seront réservées à des chercheurs français désirant se rendre dans des laboratoires étrangers et à des chercheurs étrangers venant travailler dans des laboratoires français. Elles s'adressent aux jeunes chercheurs, moins de 35 ans, et sont normalement d'une durée maximale d'un an; elles peuvent éventuellement être renouvelées.

Seules seront prises en considération les demandes de bourses qui entrent explicitement dans les objectifs de la Fondation.

\section{PRIX INTERNATIONAL}

Un PRIX INTERNATIONAL de $300000 \mathrm{~F}$ est attribué à un chercheur qui s'est distingué par une activité de recherche fondamentale qui correspond, directement ou indirectement, à l'objectif de la Fondation et qui concerne des disciplines telles que l'éthologie, la paléontologie, l'archéologie, l'anthropologie, la psychologie, l'épistémologie, la logique et les sciences du système nerveux. Il a été décerné à MM. les Professeurs A. LEROI-GOURHAN (1980), W.H. THORPE (1981), V.B. MOUNTCASTLE (1982), H.C. CONKLIN (1983), R.W. BROWN (1984), P. BUSER (1985), D. PILBEAM (1986), D; PREMACK (1987), J.C. GARDIN (1988), P.S. GOLDMAN-RAKIC (1989), J. GOODY (1990), G.A. MILLER (1991), P. RAKIC (1992), L.L. CAVALLI-SFORZA (1993), L.R. GLEITMAN (1994), W.D. HAMILTON (1995), C. RENFREW (1996), M. JOUVET (1997), A. WALKER (1998) et B. BERLIN (1999).

Discipline pour le Prix International 2001:

\section{«ÉTHOLOGIE COMPARÉE}

Les propositions de candidature doivent comporter:

- le curriculum vitæ,

- la liste des publications du candidat,

- un résumé (quatre pages maximum) du travail de recherche qui justifie l'attribution du Prix. On ne peut se porter directement candidat. La candidature doit impérativement être présentée par une personnalité scientifique reconnue et être adressée en 15 exemplaires au Secrétariat de la Fondation, 194, rue de Rivoli, 75001 PARIS.

\section{TIRÉS À PART}

P. Wolkenstein 